\title{
Acompanhamento farmacoterapêutico em pacientes idosos de uma entidade de longa permanência de São João Da Boa Vista - SP
}

\author{
Pharmacotherapeutic monitoring of elderly patients of a long stay entity in São João da Boa \\ Vista - SP
}

\author{
Robson Andrade $^{\bullet}$, Gabriel Aparecido de Carvalho* ${ }^{\bullet}$, Danyelle Marini ${ }^{\bullet}$ \\ Centro Universitário das Faculdades Associadas de Ensino. São João da Boa Vista, São Paulo, Brasil. *Autor para correspondência. E- \\ mail: Carvallhovgs@gmail.com
}

\begin{abstract}
Resumo: O acompanhamento farmacoterapêutico (AF) é um grande desafio para o profissional farmacêutico e se torna uma ferramenta importante para reduzir erros com medicações, o que interfere na eficácia do tratamento, resultando na melhora ou não dos problemas de saúde e da qualidade de vida. No caso dos idosos, a qualidade de vida é dependente da sua capacidade de junção entre saúde emocional e física, pois com o avanço da idade, sua capacidade funcional diminui, e eles tendem a perder sua independência e assim comprometendo a sua autonomia. O objetivo deste estudo foi realizar $\mathrm{o}$ acompanhamento farmacoterapêutico de idosos, afim, de amenizar erros referente ao tratamento medicamentoso, resultando na melhora da qualidade de vida destes pacientes. Material e métodos: Foi realizado uma pesquisa utilizando o Método Soap em que foram coletados os dados subjetivos, dados objetivos, em seguida realizou a avaliação e por fim, o plano de ações para o problema identificados. Resultados e discussão: Dos 10 pacientes entrevistados, as patologias mais apresentadas foram problemas cardiovasculares e diabetes mellitus, dos 10 pacientes entrevistados, em 3 não ocorreu nenhuma interação medicamentosa, porém, 7 apresentaram interações medicamentosas, referente ao seu tratamento farmacológico, sendo 32\% interações graves e $68 \%$ moderadas, Qualquer efeito indesejado que possa interferir e prejudicar a farmacoterapia do paciente é definido como Problemas Relacionados com Medicamentos (PRMs). Considerações finais: $\mathrm{O}$ acompanhamento farmacoterapêutico mostra a importância da inserção do farmacêutico na equipe multidisciplinar de saúde, em que ela possa exercer e praticar os conhecimentos adquiridos na graduação, a fim de garantir a eficiência da farmacoterapia e por fim melhorar a qualidade de vida dos pacientes.
\end{abstract}

Palavras-chave: acompanhamento farmacoterapêutico, idosos, problemas relacionados a medicamentos, qualidade de vida, SOAP.

\begin{abstract}
Pharmacotherapeutic monitoring (PA) is a major challenge for the pharmaceutical professional and becomes an important tool to reduce medication errors, which interferes with the effectiveness of treatment, resulting in the improvement or not of health problems and quality of life. In the case of the elderly, the quality of life depends on their ability to combine emotional and physical health, because with advancing age, their functional capacity decreases, and they tend to lose their independence, thus compromising their autonomy. The objective of this study was to carry out the pharmacotherapeutic followup of the elderly, in order to alleviate errors related to drug treatment, resulting in an improvement in the quality of life of these patients. Material and methods: A survey was carried out using the Soap Method in which subjective data and objective data were collected, then carried out the evaluation and finally, the action plan for the identified problem. Results and discussion: Of the 10 patients interviewed, the most common pathologies were cardiovascular problems and diabetes mellitus, of the 10 patients interviewed, in 3 there was no drug interaction, however, 7 had drug interactions, referring to their pharmacological treatment, with 32\% interactions severe and 68\% moderate. Any unwanted effect that could interfere with and impair the patient's pharmacotherapy is defined as Drug Related Problems (DRPs). Final considerations: Pharmacotherapeutic monitoring shows the importance of inserting the pharmacist in the multidisciplinary health team, in which she can exercise and practice the knowledge acquired at graduation, in order to guarantee the efficiency of pharmacotherapy and finally improve the quality of life of patients.
\end{abstract}

Keywords: pharmacotherapeutic follow-up, elderly, drug-related problems, quality of life, SOAP. 


\section{Introdução}

O envelhecimento é caracterizado por processo congênito da vida, no qual ocorrem várias mudanças desde alterações fisiológicas, motoras, psicológicas e no âmbito social (Mendes et al., 2005).

O idoso é definido pela sua idade decorrente. Nos países em desenvolvimento, a pessoa é considerada idosa a partir dos 60 anos, já em países desenvolvidos é considerado a partir dos 65 anos (Mendes et al., 2005).

Segundo o Ministério da Saúde (Brasil, 2006), o envelhecimento da população no Brasil vem tendo um aumento constante. Estima-se que o Brasil tem 20,6 milhões de idosos (IBGE, 2010).

De acordo com Cabral et al. (2013), foram identificadas que a melhoria na qualidade dos idosos está relacionada diretamente a ausência de doenças (35\%), situação financeira (25\%) e independência (40\%). De acordo com o Ministério da Saúde (Brasil, 2006), um grande desafio encontrado é ajudar o idoso a viver com a maior qualidade de vida possível tanto no âmbito familiar, como social, e fazer com que seus entes e amigos reconheçam as potencialidades e os valores dos idosos.

Com o envelhecimento ocorre várias mutações no organismo, existe o envelhecimento cerebral, cardiovascular, do sistema respiratório, sistema digestório, sistema urinário, do sistema imunológico e do sistema musculoesquelético. As alterações na homeostase do organismo interferem na sensibilidade podendo alterar os receptores nos quais se ligam os fármacos, resultando nas interações entre o fármaco, o receptor e a resposta final do efeito (Silva et al., 2012). Alguns medicamentos têm suas respostas aumentadas com o envelhecimento, e por isso devem ser administradas com cuidado, pois podem causar toxicidade (Mendes, 2005).

Diante do exposto os pacientes idosos têm uma grande probabilidade de ocorrer complicações com o uso dos medicamentos, visando diminuir o risco vários critérios estão sendo criados, a exemplo, pode citar os critérios de Beers publicado inicialmente em 1991, várias avaliações foram consideras, indicando os medicamentos e complicações que os idosos sofriam após o uso dos mesmos. Muitos dos estudos feitos, confirmaram os efeitos insuficientes dos fármacos em idosos, porém mesmo desta forma tem associação com patologias graves que geram, tais como hemorragias intestinais, confusão mental e riscos de os idosos sofrerem quedas (American Geriatrics Society, 2015).

Outro fato a ser considerado é a probabilidade de ocorrer interações medicamentosas, estas podem ser benéficas ou não, pois os medicamentos quando juntos, podem ter seu efeito terapêutico alterado, resultando na ineficácia dos mesmos, ou no aumento dos efeitos, ocasionando toxicidade ao paciente (Brasil, 2008). As alterações medicamentosas, quando tem seu efeito terapêuticos alterados, e resultam na ineficácia do fármaco, podem ser perigosas (Brasil, 2008).

Segundo Silva et at. (2010), as interações medicamentosas são classificadas conforme os riscos que elas trazem: leve quando não trazem risco ao paciente e não modificam o tratamento; moderada são aquelas que podem afetar o tratamento, trazendo um risco de piora ao paciente, elevando o nível do tratamento, fazendoo regredir; grave são interações que trazem risco na vida do paciente, necessitando de terapias adicionais para preservar a vida do paciente.

Um dos maiores problemas relacionados as interações medicamentos, é a polifarmácia (Dias, 2001; Pereira, 2008). O alto número de medicamentos prescritos para cada paciente, resulta no aumento da incidência de interações medicamentosas (Ceia, 2007). Calcula-se que a porcentagem de pacientes que sofrem com interações medicamentosas seja de 3 e $5 \%$, sendo que esses pacientes são os que fazem o uso de um número menor de fármacos, porém para pacientes que usam um número maior de medicamentos, acima de 10, essa porcentagem chega a $20 \%$ (Bustamante, 2005).

Todos os fatores expostos acima podem causar Problemas Relacionados com os Medicamentos (PRM) definido como reposta negativa ocasionada pelo tratamento farmacológico, o que permite a não atingir o objetivo terapêutico desejado ou até mesmo o aparecimento de efeitos indesejáveis (Comité de Consenso, 2002).

Visando evitar os PRM uma ferramenta de grande valor é o Acompanhamento Farmacoterapêutico que tem por objetivo melhorar a qualidade de vida do paciente por meio da prevenção e orientação, responsabilizando-se pelas necessidades do paciente ligadas aos medicamentos. Este acompanhamento zela também pela promoção à saúde, evidenciando os erros e promovendo à saúde do paciente. O acompanhamento farmacoterapêutico visa a conciliação de medicamentos ou a revisão da farmacoterapia. Há evidências de resultados positivos desta prática do farmacêutico (OPAS, 2002).

\section{Material e métodos}

O presente estudo refere a uma pesquisa transversal com 10 pacientes de um lar de idosos em São João da Boa Vista. A condução de um estudo transversal envolve algumas características e etapas, que são as 
seguintes, a saber, definição de uma população de interesse e estudo da população por meio da realização de censo ou amostragem de parte dela.

A pesquisa ocorreu em um Lar de Idosos e São João da Boa Vista. Atualmente habitam 37 idosos, os quais precisam de cuidados integrais. Foram coletados dados e algumas informações e realizado algumas entrevistas com os pacientes para proceder o acompanhamento farmacoterapêutico.

Todos os pacientes inseridos no Acompanhamento Farmacoterapêutico, residem no Lar de Idosos São José, necessitam de cuidados em período integral. Foram selecionados 10 idosos acima de 60 anos de idade, que tinham condições de responder os questionamentos e aceitaram a participar da pesquisa após a assinatura do termo livre esclarecido.

Na primeira entrevista o paciente foi informado do objetivo do Acompanhamento Farmacoterapêutico que se refere a conseguir a máxima efetividade dos medicamentos que se utiliza, que sua realização não substituí nenhum outro tratamento indicado por outro profissional de saúde em sua função, não iniciará e nem suspenderá nenhum tratamento, nem modificará nenhuma posologia prescrita pelo médico e sempre que necessário entrará em contato com ele visando melhorar tratamento farmacológico, também sensibilizou o paciente sobre sua responsabilidade e colaboração necessárias para a participação dele na tomada de decisões relacionadas ao tratamento medicamentosos.

Ressalta que, os formulários apresentados neste trabalho foram retirados dos Livros de Cuidados Farmacêuticos na Atenção Básica, publicado pelo Ministério da Saúde (Brasil, 2008).

No primeiro momento da entrevista foram coletados dados para estabelecer o perfil do paciente, como local de atendimento, nome, idade, sexo, escolaridade, ocupação, peso, altura, ICM, autonomia na gestão dos medicamentos, entre outros.

Ao encerramento da coleta de dados, foi feito uma análise de todas elas e utilizou para apontar todas os problemas na farmacoterapia do paciente, contou com o auxílio do programa Micromedex 2.0 disponibilizado no Portal Saúde Baseada em Evidências e com o Critério de Beers 2015 para identificar a presença de medicamentos inapropriados para o uso de idosos (Micromedex, 2016; American Geriatrics Society, 2015).

Após a análise estabeleceu as suspeitas de PRM que o paciente apresentava. Definiu o perfil do paciente e estabeleceu prioridades em relação ao balanço efetividade/segurança, e com isso foi possível determinar um plano de cuidado e assim realizar as intervenções farmacêuticas necessária. As que não alteram a prescrição dos medicamentos foram feitas diretamente com o farmacêutico, porém aquelas que necessitaram, foram encaminhadas ao médico (Brasil, 2006).

\section{Resultados e Discussão}

Esta pesquisa abordou 10 pacientes residentes em uma entidade de longa permanência de São João da Boa Vista - SP. Estes pacientes eram compostos por mulheres (50\%) homens (50\%).

No que se refere ao grau de escolaridade, foi notado que o maior percentual de escolaridade foi dos homens, sendo ao ensino fundamental (60\%), médio (20\%) e superior (20\%). Já para as mulheres, todas as pacientes relataram somente tinham o ensino fundamental (100\%) (Tabela 1).

Tabela 1. Distribuição de entrevistados segundo a escolaridade.

\begin{tabular}{ccccccc}
\hline \multirow{2}{*}{ Nível de escolaridade } & \multicolumn{2}{c}{ Homens } & \multicolumn{2}{c}{ Mulheres } & \multicolumn{2}{c}{ Total } \\
\cline { 2 - 7 } & $\mathrm{n}$ & $\%$ & $\mathrm{n}$ & $\%$ & $\mathrm{n}$ & $\%$ \\
\hline Superior Completo & - & - & - & - & - & - \\
Superior Incompleto & 1 & 20 & & & 1 & 10 \\
Médio Completo & 1 & 20 & - & - & 1 & 10 \\
Médio Incompleto & & & & & - & - \\
Fundamental Completo & - & - & 1 & 20 & 1 & 10 \\
Fundamental Incompleto & 3 & 60 & 4 & 80 & 7 & 70 \\
\hline TOTAL & 5 & 100 & 5 & 100 & 10 & 100 \\
\hline
\end{tabular}

No que se refere a faixa etária, todos os pacientes tinham suas idades acima de 60 anos, caracterizando os idosos (Tabela 2).

No início das consultas, os 10 pacientes foram questionados sobre a Qualidade de Vida e a Percepção Geral da Saúde, em que eles deveriam se autoavaliar. Dos 10 pacientes, 4 deram a nota 8, relatando que são felizes, 
porém relatam que suas patologias interferem na sua qualidade de vida, 3 deram nota 7 , justificando as patologias, dificuldades de locomoção como interferências em suas vidas. Já 2 relataram nota 10, justificando com a boa vida mesmo com as patologias e problemas existentes em decorrer da idade.

Dos 10 pacientes entrevistados, as patologias mais apresentadas foram problemas cardiovasculares presente em 7 idosos e diabetes mellitus em 4 idosos (Figura 1).

Tabela 2. Distribuição de entrevistados segundo a faixa etária.

\begin{tabular}{ccccccc}
\hline \multirow{2}{*}{ Faixa etária } & \multicolumn{2}{c}{ Homens } & \multicolumn{2}{c}{ Mulheres } & \multicolumn{2}{c}{ Total } \\
\cline { 2 - 7 } & $\mathrm{n}$ & $\%$ & $\mathrm{n}$ & $\%$ & $\mathrm{n}$ & $\%$ \\
\hline $60-65$ & - & - & - & - & - & - \\
$66-70$ & 2 & 40 & 1 & 20 & 3 & 30 \\
$71-75$ & - & - & - & - & - & - \\
$76-80$ & 1 & 20 & 3 & 60 & 4 & 40 \\
$81-85$ & 1 & 20 & 1 & 20 & 2 & 20 \\
$86-90$ & 1 & 20 & - & - & 1 & 10 \\
\hline TOTAL & 5 & 100 & 5 & 100 & 10 & 100 \\
\hline
\end{tabular}

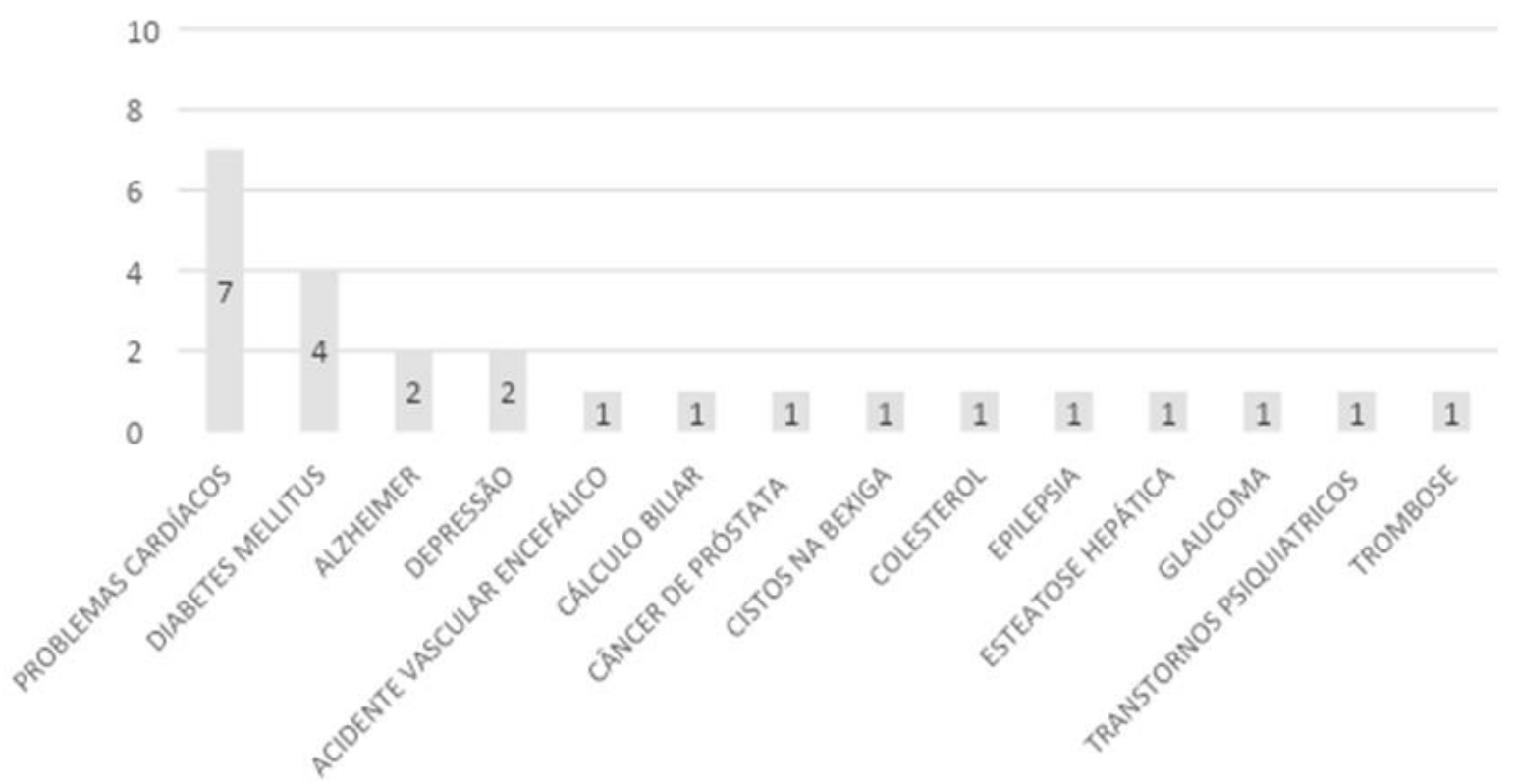

Figura 1. Distribuição de patologias segundo a quantidade de idosos que apresentam cada uma delas. Fonte: Autores (2017).

Os pacientes são atendidos na UBS do próprio bairro de residência dos idosos e os medicamentos eram adquiridos em sua maioria na própria unidade de saúde e nas redes de farmácia popular. A tabela 3 relaciona os medicamentos prescritos aos idosos, organizando-os pela classificação ATC.

Dos 10 pacientes entrevistados, em 3 não ocorreu nenhuma interação medicamentosa, porém, 7 apresentaram interações medicamentosas, referente ao seu tratamento farmacológico totalizando 25 interações, sendo 8 (32\%) interações graves e 17 (68\%) moderadas.

No que se refere a interações moderadas, são aquelas que podem trazer piora no quadro de vida do paciente, necessitando de alterações no tratamento, foram encontradas duas vezes as interações entre metformina e propranolol, carbonato de cálcio, vitamina d e hidroclorotiazida, carbonato de cálcio, vitamina d e propranolol e hidroclorotiazida e propranolol (Tabela 4).

Das prescrições analisadas, verificou que ocorreu 8 interações medicamentosas graves, que são aquelas que trazem risco de vida ao paciente, requerendo intervenção médica. Vale destacar que as oito interações encontradas ocorreram somente uma vez (Tabela 5). 
Tabela 3. Medicamentos prescritos de acordo com a classificação ATC.

\begin{tabular}{|c|c|c|c|}
\hline ATC & Medicamento & $\mathbf{n}$ & $\%$ \\
\hline A02BC01 & omeprazol cápsula 20 mg & 5 & $11,62 \%$ \\
\hline A03FA03 & domperidona comprimido $10 \mathrm{mg}$ & 1 & $2,32 \%$ \\
\hline A10BA02 & metformina $500 \mathrm{mg}$ & 2 & $4,65 \%$ \\
\hline A10BA02 & metformina $850 \mathrm{mg}$ & 1 & $2,32 \%$ \\
\hline A12AA04 & carbonato de cálcio 500mg & 2 & $4,65 \%$ \\
\hline B01AC04 & clopidogrel comprimido $75 \mathrm{mg}$ & 1 & $2,32 \%$ \\
\hline B01AC06 & ácido acetilsalicílico 100 mg & 1 & $2,32 \%$ \\
\hline B01AC23 & cilostazol comprimido $100 \mathrm{mg}$ & 1 & $2,32 \%$ \\
\hline C01AA05 & digoxina comprimido $0,25 \mathrm{mg}$ & 1 & $2,32 \%$ \\
\hline C01BD01 & amiodarona comprimido $200 \mathrm{mg}$ & 1 & $2,32 \%$ \\
\hline C03AA03 & hidroclorotiazida comprimido $25 \mathrm{mg}$ & 2 & $4,65 \%$ \\
\hline C03CA01 & furosemida comprimido $40 \mathrm{mg}$ & 1 & $2,32 \%$ \\
\hline C03DA01 & espirolactona comprimido $25 \mathrm{mg}$ & 1 & $2,32 \%$ \\
\hline C04AD03 & pentoxifilina comprimido $400 \mathrm{mg}$ & 2 & $4,65 \%$ \\
\hline C07AA05 & propranolol comprimido $40 \mathrm{mg}$ & 2 & $4,65 \%$ \\
\hline C08CA01 & anlodipino besilato comprimido $5 \mathrm{mg}$ & 1 & $2,32 \%$ \\
\hline C08CA05 & nifedipina cpr. de liberação lenta 20 mg & 2 & $4,65 \%$ \\
\hline C09CA01 & losartana comprimido $50 \mathrm{mg}$ & 3 & $6,97 \%$ \\
\hline C10AA01 & sinvastatina comprimido revestido $20 \mathrm{mg}$ & 1 & $2,32 \%$ \\
\hline L02BB03 & bicalutamida comprimido revestido $50 \mathrm{mg}$ & 1 & $2,32 \%$ \\
\hline M05BA04 & alendronato de sódio comprimido $70 \mathrm{mg}$ & 1 & $2,32 \%$ \\
\hline N02AX02 & tramadol cápsula 50 mg & 1 & $2,32 \%$ \\
\hline N03AA02 & fenobarbital comprimido $100 \mathrm{mg}$ & 1 & $2,32 \%$ \\
\hline N03AB02 & fenitoína comprimido $100 \mathrm{mg}$ & 2 & $4,65 \%$ \\
\hline N05AD01 & haloperidol comprimido $5 \mathrm{mg}$ & 1 & $2,32 \%$ \\
\hline N05BA01 & diazepam comprimido $10 \mathrm{mg}$ & 1 & $2,32 \%$ \\
\hline N06DA02 & donepezila comprimido $10 \mathrm{mg}$ & 1 & $2,32 \%$ \\
\hline N06DA03 & rivastigmina comprimido $6 \mathrm{mg}$ & 1 & $2,32 \%$ \\
\hline R05CB01 & acetilcisteína pó granulado 200mg & 1 & $2,32 \%$ \\
\hline S01ED01 & timolol solução oftálmica $0,25 \%$ & 1 & $2,32 \%$ \\
\hline TOTAL & & 43 & $100 \%$ \\
\hline
\end{tabular}

Tabela 4. Distribuição dos pacientes segundo a presença de interações moderadas nas prescrições.

\begin{tabular}{|c|c|c|c|}
\hline Medicamentos & n & $\%$ & Consequência da interação \\
\hline metformina e propranolol & 2 & $11,7 \%$ & Pode causar um quadro de hipoglicemia \\
\hline timolol e metformina & 1 & $5,8 \%$ & $\begin{array}{l}\text { Pode aumentar ou diminuir o efeito de redução da glicemia no agente } \\
\text { antidiabético }\end{array}$ \\
\hline amiodarona e losartana & 1 & $5,8 \%$ & $\begin{array}{l}\text { Pode resultar em níveis plasmáticos aumentados de losartana e níveis } \\
\text { plasmáticos diminuídos do metabolito ativo. }\end{array}$ \\
\hline amiodarona e propranolol & 1 & $5,8 \%$ & $\begin{array}{l}\text { Pode resultar em efeitos cardíacos aditivos (por exemplo, bradicardia, prisão } \\
\text { de sinus e bloqueio } \mathrm{AV} \text { ). }\end{array}$ \\
\hline $\begin{array}{l}\text { carbonato de cálcio, vitamina d e } \\
\text { hidroclorotiazida }\end{array}$ & 2 & $11,7 \%$ & Pode resultar em hipercalcemia. \\
\hline $\begin{array}{l}\text { carbonato de cálcio, vitamina d e } \\
\text { propranolol }\end{array}$ & 2 & $11,7 \%$ & Pode resultar na diminuição da biodisponibilidade do propranolol. \\
\hline furosemida e propranolol & 1 & $5,8 \%$ & Pode resultar em hipotensão e bradicardia. \\
\hline hidroclorotiazida e propranolol & 2 & $11,7 \%$ & Pode resultar em hiperglicemia, hipertrigliceridemia. \\
\hline hidrocloriotiazida e prednisona & 1 & $5,8 \%$ & Pode resultar em hipocalemia e subsequentes arritmias cardíacas. \\
\hline omeprazol e propranolol & 1 & $5,8 \%$ & Pode resultar em aumento da exposição ao propranolol. \\
\hline prednisona e propranolol & 1 & $5,8 \%$ & $\begin{array}{l}\text { Pode levar à diminuição das concentrações de propranolol, resultando em } \\
\text { perda de eficácia. }\end{array}$ \\
\hline losartana e espirolactona & 1 & $5,8 \%$ & Pode resultar em hipercalemia. \\
\hline omeprazol e fenitoína & 1 & $5,8 \%$ & $\begin{array}{l}\text { Pode resultar em um risco aumentado de toxicidade de fenitoína (ataxia, } \\
\text { hiperreflexia, nistagmo, tremor). }\end{array}$ \\
\hline TOTAL & 17 & $100 \%$ & - \\
\hline
\end{tabular}


Tabela 5. Distribuição dos pacientes segundo a presença de interações graves nas prescrições.

\begin{tabular}{|c|c|c|c|}
\hline Medicamentos & $\mathbf{n}$ & $\%$ & Consequência da interação \\
\hline omeprazol e clopidogrel & 1 & $12,5 \%$ & Diminui a eficácia do clopidogrel e com isso diminui a agregação plaquetária. \\
\hline amiodarona e nifedipino & 1 & $12,5 \%$ & Aumento do nível ou o efeito da amiodarona. \\
\hline amiodarona e sinvastatina & 1 & $12,5 \%$ & Aumento da exposição à sinvastatina e aumento do risco de miopatia ou rabdomiólise. \\
\hline captopril e digoxina & 1 & $12,5 \%$ & Aumento da concentração plasmática de digoxina. \\
\hline digoxina e fenitoína & 1 & $12,5 \%$ & A coadministração pode diminuir as concentrações plasmáticas de digoxina. \\
\hline nifedipino e prednisona & 1 & $12,5 \%$ & Pode reduzir significativamente a eficácia da nifedipino. \\
\hline diapezam e fenitoína & 1 & $12,5 \%$ & Alteração na concentração sérica de fenitoína. \\
\hline cilostazol e omeprazol & 1 & $12,5 \%$ & Aumento da exposição ao cilostazol. \\
\hline TOTAL & 8 & $100 \%$ & \\
\hline
\end{tabular}

De acordo com os Critérios de Beers, publicado pela Sociedade de Geriatria Americana, os medicamentos inapropriados para idosos devem ser evitados por serem propícios a trazerem problemas e complicações na saúde dos idosos. Das prescrições analisadas, foi verificado o uso de 6 medicamentos classificados por estes critérios. O medicamento omeprazol foi o mais prescrito para cinco idosos (Tabela 6).

Tabela 6. Distribuição dos medicamentos inapropriados segundo o critério de Beers.

\begin{tabular}{|c|c|c|}
\hline Medicamento & $\mathbf{n}$ & Racionalidade \\
\hline omeprazol & 5 & $\begin{array}{l}\text { Risco de infecções por Clostridium difficile e perda óssea, resultando em } \\
\text { fraturas. }\end{array}$ \\
\hline fenobarbital & 1 & $\begin{array}{c}\text { Alta taxa de dependência física, tolerância aos benefícios do sono, maior } \\
\text { risco de overdose em baixas doses }\end{array}$ \\
\hline nifedipino & 2 & Potencial de hipotensão; risco de precipitação da isquemia miocárdica \\
\hline diazepam & 1 & $\begin{array}{c}\text { Aumentam o risco de comprometimento cognitivo, delírio, quedas, } \\
\text { fraturas e acidentes de veículos em idosos }\end{array}$ \\
\hline amiodarona & 1 & $\begin{array}{l}\text { É mais tóxica do que antiarrítmicos utilizados na fibrilação atrial. Pode } \\
\text { ser uma terapia de escolha de primeira linha razoável em pacientes com } \\
\text { insuficiência cardíaca concomitante ou hipertrofia ventricular esquerda. }\end{array}$ \\
\hline digoxina & 1 & $\begin{array}{c}\text { Não deve ser usado como agente de primeira escolha em fibrilação atrial, } \\
\text { pois podem estar associados ao aumento da mortalidade e existem } \\
\text { alternativas mais efetivas para serem usadas. }\end{array}$ \\
\hline
\end{tabular}

Qualquer efeito indesejado que possa interferir e prejudicar a farmacoterapia do paciente é definido como Problemas Relacionados com Medicamentos (PRMs). Dos 10 pacientes analisados, 8 apresentaram. Dos PRMs encontrados, 3 são de necessidade (por apresentar patologias não tratadas) e 14 de insegurança (sendo 11 por medicamentos contraindicados, já que os pacientes fazem uso de medicamentos inapropriados de acordo com os critérios de Beers, 1 por apresentar reação adversa ao medicamento e 2 por interações medicamentosas, onde trazem prejuízos a terapia) (Tabela 7).

Tabela 7. Classificação dos problemas relacionados aos medicamentos.

\begin{tabular}{cccc}
\hline PRM & Tipo & $\mathbf{n}$ & $\mathbf{\%}$ \\
\hline Necessidade & Problema de saúde não tratado & 3 & 17,64 \\
\hline \multirow{3}{*}{ Insegurança } & Medicamentos contraindicados & 11 & 64,70 \\
& Reações adversas & 1 & 5,88 \\
& Interação medicamentosa & 2 & 11,76 \\
\hline TOTAL & 17 & 100 & \\
\hline
\end{tabular}

Devido as interações medicamentosas e problemas relacionados aos medicamentos encontrados, 4 dos 10 pacientes precisaram de intervenção, sendo utilizada o modelo de carta ao médico. Nesta carta foram apresentados os dados do paciente, terapia que o paciente utiliza, os problemas apresentados perante o estudo farmacoterapêutico e as possíveis soluções para os problemas apresentados. A sugestão nestes casos, foram as trocas dos medicamentos, por medicamentos com menos reações adversas e mais efetivas, além de possível encaminhamento ao profissional psicólogo, no caso da paciente que apresenta depressão. 


\section{Considerações finais}

Este presente estudo possibilitou realizar o acompanhamento farmacoterapêutico em pacientes idosos. Este tipo de paciente normalmente apresenta várias patologias crônicas que são associadas a senescência, resultando ao uso de vários medicamentos de diversas classes terapêuticas. Conforme visto, foi constatado o uso de medicamentos inapropriados aos idosos de acordo com os critérios de Beers, além do uso concomitante de medicamentos que ocasionam interações medicamentosas, resultando em problemas relacionados aos medicamentos os quais podem prejudicar uma farmacoterapia eficiente. Aos pacientes que precisaram de intervenção medicamentosa, foi enviado ao médico prescritor uma carta com uma possível solução do problema encontrado, além de encaminhamentos a outros profissionais da saúde, tal como o psicólogo.

O acompanhamento farmacoterapêutico mostra a importância da inserção do farmacêutico na equipe multidisciplinar de saúde, em que ela possa exercer e praticar os conhecimentos adquiridos na graduação, a fim de garantir a eficiência da farmacoterapia e por fim melhorar a qualidade de vida dos pacientes.

\section{Referências}

American Geriatrics Society. 2015. American Geriatrics Society 2015 Updated Beers Criteria for Potentially Inappropriate Medication Use in Older Adults. Journal of the American Geriatrics Society, 63(11), 2227-2246.

Brasil. Ministério da Saúde. 2006. Secretaria de Atenção a Saúde. Departamento de Atenção Básica. Envelhecimento e saúde da pessoa idosa. Cadernos de Atenção Básica. Brasília: Ministério da Saúde, 192p.

Brasil. Ministério da Saúde. 2008. Secretaria de Ciência, Tecnologia e Insumos Estratégicos. Departamento de Assistência Farmacêutica e Insumos Estratégicos. Formulário terapêutico nacional 2008: Rename 2006. Brasília: Ministério da Saúde, 897p.: il. - (Série B. Textos Básicos de Saúde).

Bustamante, G. D. D., Cabrera, C., Duran, G. M. G., \& Nunez, M. T. J. 2005. Detección de interacciones medicamentosas, em pacientes ingresados a la unidad de cuidados intensivos del Instituto Autônomo Hospital Universitário de los Andes. Vitae Academia Biomédica Digita, 25(7), 1-16.

Cabral, R. W. L., Santos, S. R., Menezes, K. D. N. B., Albuquerque, A. V., \& Medeiros, A. L. 2013. Fatores sociais e melhoria da qualidade de vida dos idosos: revisão sistemática. Revista de Enfermagem UFPE On Line, 5(7), 1434-1442, 2013.

Ceia, F. 2007. Interações medicamentosas na prática clínica. Revista Portuguesa de Clínica Geral, 23(2), 197 -207.

Dias, M.F. 2001. Interações medicamentosas potenciais em ambiente hospitalar. Dissertação, Faculdade de Ciências Médicas, Universidade Estadual de Campinas, Campinas.

IBGE. Instituto Brasileiro de Geografia e Estatística. Censo 2010. Disponível em: <http://censo2010.ibge.gov.br/>. Acesso em 15 de agosto de 2017.

Mendes, M. R. S. S. B., Gusmão, J. L., Faro, A. C. M., \& Leite, R. C. B. O. 2005. A situação social do idoso no Brasil: uma breve consideração. Acta Paulista de Enfermagem, 18(4), 422-426.

Micromedex. 2016. Portal da Saúde baseado em evidências. Disponível em: <psbe.ufrn.br > Página Principal > bases de dados>. Acesso em 07 março de 2017

OPAS. Organização Pan-Americana Da Saúde. 2002. Consenso Brasileiro de Atenção Farmacêutica: proposta. Brasília: Opas.

Comité de Consenso. 2002. Segundo consenso de Granada sobre problemas relacionados con medicamentos. Ars Pharm., 43(3-4), 175-184.

Pereira, G. J. S. 2008. Estudo de utilização de medicamentos na clínica médica. Revista Brasileira de Farmácia, 89(3), 267-271.

Silva, N. M. O., Carvalho, R. P., Bernardes, A. C. A., Moriel, P., Mazzola, P. G., \& Franchini, C. C. 2010. Avaliação de Potenciais Interações Medicamentosas de Pacientes Internados, em Hospital Público Universitário Especializado em Saúde da Mulher, em Campinas - SP. Revista Ciências Farmacêuticas Básica e Aplicada, 31(2), 171-176.

Silva, R., Schmidt, O. F., \& Silva, S. 2012. Polifarmácia em geriatria. Revista da AMRIGS, 56(2), 164-174.

\section{Minicurrículo}

Robson Andrade. Graduado em Farmácia pelo Centro Universitário das Faculdades Associadas de EnsinoFAE. 
Gabriel Carvalho. Graduando em Farmácia pelo Centro Universitário das Faculdades Associadas de EnsinoFAE.

Danyelle Marini. Doutora em Educação pela Universidade Metodista de Piracicaba (UNIMEP); Mestre em Biologia Celular e Molecular pelas Universidade Júlio Mesquita de São Paulo (UNESP); Especialista em Docência do Ensino Superior pela Gama Filho; Especialista em Cosmetologia pela UNIMEP; Graduada em Farmácia Bioquímica pela UNIMEP. Atua como docente e Coordenadora nas Faculdades Integradas Maria Imaculada; Docente do curso de graduação em Farmácia e do Mestrado do Centro Universitário das Faculdades Associadas de Ensino (UNIFAE), presidente e Presidente do Comitê de Ética do UNIFAE; Conselheira pelo Conselho Regional de Farmácia do Estado de São Paulo (CRF-SP); Membro do Comitê de Educação Permanente do CRF-SP e da Comissão de Educação do CRF-SP.

Como citar: Andrade, R., Carvalho, G., \& Marini, D. 2022. Acompanhamento farmacoterapêutico em pacientes idosos de uma entidade de longa permanência de São João Da Boa Vista - $\quad$ SP. Pubsaúde, 8, a238. DOI: https://dx.doi.org/10.31533/pubsaude8.a238

Recebido: 07 ago. 2021.

Revisado e aceito: 29 jan. 2022.

Conflito de interesse: os autores declaram, em relação aos produtos e companhias descritos nesse artigo, não ter interesses associativos, comerciais, de propriedade ou financeiros que representem conflito de interesse.

Licenciamento: Este artigo é publicado na modalidade Acesso Aberto sob a licença Creative Commons Atribuição 4.0 (CC-BY 4.0). 